\title{
THE HYPERQUASICENTER OF A FINITE GROUP. II
}

\author{
N. P. MUKHERJEE
}

\begin{abstract}
The role of Theorem B of Hall and Higman has been explained in detail to complete the proof of the fact that the hyperquasicenter is the largest supersolvably immersed subgroup. Other results included contain some sufficient conditions for supersolvability of a group and for the nontriviality of its center.
\end{abstract}

I. The proof of Theorem 1.2 in The hyperquasicenter of a finite group. I (Proc. Amer. Math. Soc. 26 (1970)) needs to be elaborated. One needs to consider only the case when $r$ is positive and is a prime and induction may be applied to show that $x^{r}$ is a $p$-group. Once this is done the proof may be completed by breaking it up into two cases: (i) $r=p$. In this case the proof may be completed by induction if $|x|$ is divisible by a prime different from $p$, or else one needs to consider the case when $G=\langle x\rangle\langle y\rangle$ is a $p$-group. In this case the proof may be completed as given in the paper by taking $Z$ to be a subgroup of order $p$. (ii) $r \neq p$. In this case one needs to use Ore's theorem [1, p. 438, Theorem 16] namely that every quasinormal subgroup of a group is subnormal.

In this connection, it may be said that it was brought to the author's notice that the proofs of Theorems 1.2 and 1.8 needed to be modified. These imperfections are attributable to complete breakdown of communications between the author and the referee. The proof of Theorem 1.8 follows from Theorem 6.1 of Baer in [4], and that requires that $G$ induces in every chief factor contained in $P$, a Sylow $p$-subgroup of the quasicenter, an abelian group of automorphisms. The verification of the fact that $G$ does satisfy this requirement is crucially dependent on the well-known Theorem B of Hall and Higman in [2]. Several other results have been included without proof. They are not difficult to verify.

II.

THEOREM. If $x$ is a $Q C$-element of a group $G$ then $x^{r}$ is also a $Q C$-element of $G$ for every integer $r$.

Proof. It is evident that it is sufficient to prove the theorem when $r$ is positive and $r$ is a prime. Let $y$ be an arbitrary but fixed element of $G$. Then

Received by the editors January 28, 1970 and March 2, 1970 and, in revised form, December 23, 1970.

AMS 1969 subject classifications. Primary 2040.

Key words and phrases. Quasicentral element (QC-element), quasicenter, hyperquasicenter.

(c) American Mathematical Society 1972 
we must prove $\left\langle x^{r}\right\rangle\langle y\rangle=\langle y\rangle\left\langle x^{r}\right\rangle$ for all $r$. The proof is by induction on $|G|$ and so we need only consider the case when $G=\langle x\rangle\langle y\rangle=\langle y\rangle\langle x\rangle$. If $|y|=$ $p_{1}^{a_{1}} \cdot p_{2}^{a_{2}} \cdots p_{n}^{a_{n}}$ where $p_{i}$ for $i=1,2, \cdots, n$ is a prime then $\langle y\rangle=$ $\left\langle y_{1}\right\rangle \times\left\langle y_{2}\right\rangle \times \cdots \times\left\langle y_{n}\right\rangle$ where $y_{i}$ is a power of $y$ and $\left|y_{i}\right|=p_{i}^{a_{i}}$. Then if $\left\langle x^{r}\right\rangle\left\langle y_{i}\right\rangle=\left\langle y_{i}\right\rangle\left\langle x^{r}\right\rangle \forall i$, we will have $\left\langle x^{r}\right\rangle\langle y\rangle=\langle y\rangle\left\langle x^{r}\right\rangle$. So we need only consider the case when $y$ is a $p$-element and $G=\langle x\rangle\langle y\rangle=\langle y\rangle\langle x\rangle$. Let $|y|=p^{b}$. If $|x|=p^{a} q,(p, q)=1$ then $\langle x\rangle=\left\langle x_{1}\right\rangle \times\left\langle x_{2}\right\rangle$ where $x_{i}$, for $i=1,2$, is a power of $x$ and $\left|\left\langle x_{1}\right\rangle\right|=p^{a}$ and $\left|\left\langle x_{2}\right\rangle\right|=q$. Since a quasinormal subgroup of a group is subnormal [1, p. 438, Theorem 16] it follows that $\langle x\rangle$ is subnormal in $G$. If $\langle x\rangle$ is normal in $G$ then $\left\langle x^{r}\right\rangle \triangleleft G$ and so $\left\langle x^{r}\right\rangle\langle y\rangle=\langle y\rangle\left\langle x^{r}\right\rangle$. Otherwise we have a subnormal chain as follows: $\langle x\rangle \triangleleft \triangleleft \triangleleft \cdots \triangleleft H \triangleleft G$. Since $x \in H$ and any element in $H$ can be written as $x^{m} y^{n}$ where $m, n$ are integers it follows that $\left\{y^{n} \mid x^{m} y^{n} \in H\right\}$ is a subgroup $\left\langle y^{d}\right\rangle$ of $\langle y\rangle$. Hence $H \subseteq\langle x\rangle\left\langle y^{d}\right\rangle$ and so $H=$ $\left\langle y^{d}\right\rangle\langle x\rangle=\langle x\rangle\left\langle y^{d}\right\rangle$ since $H$ is a group.

Induction applies to $H$ and it follows that $x_{2}=x^{p^{a}}$ is a QC-element of $H$. We claim that $\left\langle x_{2}\right\rangle \triangleleft H$. Otherwise $T=\left\langle x_{2}\right\rangle^{d} \neq\left\langle x_{2}\right\rangle$ is a cyclic subgroup of $H$ of order $q$. But $x_{2}$ is a QC-element of $H$ and so it follows that $\left\langle x_{2}\right\rangle \cdot T=$ $T \cdot\left\langle x_{2}\right\rangle=R$ is a subgroup of $H$ and $|R|=|T|\left|\left\langle x_{2}\right\rangle\right| /\left|T \cap\left\langle x_{2}\right\rangle\right|=q \cdot v$ where $v$ is a divisor of $q$ different from 1 . Since $(p, q)=1$ it follows that $(p, v)=1$. But $R$ being a subgroup of $H,|R|$ must divide $|H|$ which is impossible since $|H|=p^{c} q$ for some integer $c$ and $v$ does not divide $p$. Hence $\left\langle x_{\mathbf{2}}\right\rangle \triangleleft H$. Since $\left|x_{2}\right|$ is prime to $p$, every Sylow subgroup of $\left\langle x_{2}\right\rangle$ is also a Sylow sugbroup of $H$ and is therefore normal in $H$. This implies that $\left\langle x_{2}\right\rangle$ is characteristic in $H$ Therefore, $\left\langle x_{2}\right\rangle \triangleleft G$ since $H \triangleleft G$.

Suppose now $r=p$. Since $\langle x\rangle=\left\langle x_{1}\right\rangle \times\left\langle x_{2}\right\rangle$ it follows that $\left\langle x^{p}\right\rangle=\left\langle x_{1}^{p}\right\rangle \times\left\langle x_{2}\right\rangle$. If $\left\langle x_{2}\right\rangle \neq 1$ then induction applies to $\bar{G}=G /\left\langle x_{2}\right\rangle$ and we have $\left\langle\bar{x}_{1}^{p}\right\rangle\langle\bar{y}\rangle=$ $\langle\bar{y}\rangle\left\langle\bar{x}_{1}^{p}\right\rangle$, where $\bar{x}_{1}^{p}=\left\langle x_{2}\right\rangle \cdot x_{1}^{p}$ and $\bar{y}=\left\langle x_{2}\right\rangle \cdot y$. This equation yields $\left\langle x_{2}\right\rangle\left\langle x_{1}^{p}\right\rangle\langle y\rangle=\left\langle x_{2}\right\rangle\langle y\rangle\left\langle x_{1}^{p}\right\rangle$, i.e. $\left\langle x^{p}\right\rangle\langle y\rangle=\langle y\rangle\left\langle x^{p}\right\rangle$. If $\left\langle x_{2}\right\rangle=1$ then $G=\langle x\rangle\langle y\rangle$ is a $p$-group. In this case the proof may be completed by repeating the arguments in Theorem 1.2 of [3] by taking $Z$ to be a subgroup of order $p$.

Suppose $r \neq p$. If $\left\langle x_{2}\right\rangle=1$ then $\langle x\rangle$ is a $p$-group and $\left\langle x^{r}\right\rangle=\langle x\rangle$. Therefore $x^{r}$ is a quasicentral element in this case. Assume therefore $\left\langle x_{\mathbf{2}}\right\rangle \neq 1$. If $r$ is prime to $q$ then also $\left\langle x^{r}\right\rangle=\left\langle x_{1}\right\rangle \times\left\langle x_{2}\right\rangle=\langle x\rangle$ and $x^{r}$ is a quasicentral element.

Let $r=q_{1}$ where $q_{1}$ is a prime divisor of $q$. Since $\left\langle x^{r}\right\rangle=\left\langle x_{1}\right\rangle \times\left\langle x_{2}^{a_{1}}\right\rangle$ it follows that if $\left\langle x_{2}^{q_{1}}\right\rangle \neq 1$ then induction applies to $G \mid\left\langle x_{2}^{q_{1}}\right\rangle$ and the proof may be completed as before. If $\left\langle x_{2}^{q_{1}}\right\rangle=1$, i.e., $\left\langle x^{r}\right\rangle=\left\langle x_{1}\right\rangle$ then we proceed as follows.

Since $\langle x\rangle$ is subnormal in $G$ it follows that $\left\langle x_{1}\right\rangle=\left\langle x^{r}\right\rangle$ is subnormal in $G$ and so we have a chain as follows: $\left\langle x^{r}\right\rangle \triangleleft\langle x\rangle \triangleleft X_{1} \triangleleft X_{2} \triangleleft \cdots \triangleleft X_{k}=G$. Now, $\left\langle x^{r}\right\rangle=O_{p}(\langle x\rangle)$ implies that $\left\langle x^{r}\right\rangle \triangleleft X_{1}$ and so $\left\langle x^{r}\right\rangle \subseteq O_{p}\left(X_{1}\right)$. Assume $\left\langle x^{r}\right\rangle \subseteq$ $O_{p}\left(X_{i}\right)$ and since $O_{p}\left(X_{i}\right) \triangleleft X_{i+1}$ it follows that $O_{p}\left(X_{i}\right) \subseteq O_{p}\left(X_{i+1}\right)$. Hence $\left\langle x^{r}\right\rangle \subseteq O_{p}\left(X_{i+1}\right)$ and so $\left\langle x^{r}\right\rangle \subseteq O_{p}\left(X_{k}\right)=O_{p}(G)$. Set $M=O_{p}(G)\langle y\rangle$. Since 
$\langle x\rangle\langle y\rangle \supseteq M \supseteq\langle y\rangle, M=(\langle x\rangle \cap M)\langle y\rangle$. But $\left\langle x^{r}\right\rangle \subseteq\langle x\rangle \cap M \neq\langle x\rangle$ and therefore $\left\langle x^{r}\right\rangle=\langle x\rangle \cap M$. This implies that $\left\langle x^{r}\right\rangle\langle y\rangle=M$ is a group and the theroem is proved completely.

It is natural to ask whether the nontriviality of the quasicenter guarantees the nontriviality of the center under suitable conditions. The following theorems provide some answers.

THEOREM. If the smallest prime divisor of the order of a group divides the order of its quasicenter then $G$ has a nontrivial center.

LEMMA. If $\bar{P}_{m}$ is a normal Sylow $p_{m}$-subgroup of a group $G$ and $p_{m}$ divides the order of the quasicenter then the quasicenter has a cyclic normal subgroup of order $p_{m}$.

THEOREM. If the smallest prime divisor of the order of a group $G$ divides the order of the hyperquasicenter and the corresponding Sylow subgroup of $G$ is normal then $G$ has a nontrivial center.

III. It has been shown that the hyperquasicenter $Q^{*}(G)$ of a group $G$ contains the largest supersolvably immersed subgroup of $G$. The proof is easy. It uses induction on $G$ and depends on a lemma that the hyperquasicenter of $G / N$ where $N \triangleleft G$ and $N \subseteq Q^{*}(G)$ is $Q^{*}(G) / N$. The proof of the lemma is quite straightforward. To prove $Q^{*}(G)$ is indeed supersolvably immersed in $G$ we need the help of Theorem 6.1 of Baer in [4].

THEOREM. The hyperquasicenter $Q^{*}(G)$ of a group $G$ is the largest supersolvably immersed subgroup of $G$.

Proof. The fact that every supersolvably immersed subgroup of $G$ is contained in $Q^{*}(G)$ has been proved in detail in Theorem 1.8 in [3]. To prove $Q^{*}(G)$ is supersolvably immersed in $G$ we proceed by induction on $|G|$. Let $Q_{p}$ be a Sylow $p$-subgroup of the quasicenter $Q(G)$ of $G$ and let $N$ be a minimal normal subgroup of $G$ contained in $Q_{p}$. It may be observed that it is enough to prove that $N$ is supersolvably immersed in $G$. For, then the result will follow by induction. To prove that $N$ is supersolvably immersed in $G$ we shall take the help of Theorem 6.1 in [4] which states that the following are equivalent:

1. $N$ is supersolvably immersed in $G$.

2. Every chief factor of $G$ contained in $N$ is cyclic of order a prime.

3. $G$ induces in every chief factor contained in $N$ an abelian group of automorphisms and $x^{p-1} \circ y=1$ for every prime $p$, every $p$-element $y$ in $N \cap G^{\prime}$ and every element $x$ in $G$ with $\left(\left|x C_{G}\left(N \cap G^{\prime}\right)\right|, p\right)=1$.

Thus we need to prove 3 and for that matter it may be noted that it is enough to prove that $G / C_{G}(N)$ is abelian and is of exponent dividing $p-1$. (Note that $N$ is elementary abelian.) 
Let $G_{1}=G / C$ and $T=C_{G}(N)$ where $C=C_{G}\left(Q_{p}\right)$. Then $C \subseteq T$ and $G_{1} / T_{1}$, where $T_{1}=T / C$ can be regarded as the group of automorphisms induced in $N$ by $G$. It may be observed that $O_{p}\left(G_{1}\right)$ is contained in $T_{1}$. For otherwise $O_{p}\left(G_{1}\right) \cdot T_{1} / T_{1}$ will be a normal $p$-group of automorphisms of $N$ and considering $N$ as a vector space over $\mathrm{GF}(p)$ we observe that since $G_{1} / T_{1}$ has a faithful irreducible representation on $N$ this is impossible. Hence $O_{p}\left(G_{1}\right)$ must necessarily be contained in $T_{1}$ which implies that in order to prove $G_{1} / T_{1}$ is abelian and is of exponent dividing $p-1$ it is enough to show that $G_{1} / O_{p}\left(G_{1}\right)$ possesses the same properties.

Let $\bar{G}=G / \Phi\left(Q_{p}\right), C^{*}=C_{G}\left(Q_{p} / \Phi\left(Q_{p}\right)\right)$ and $\bar{Q}_{p}=Q_{p} / \Phi\left(Q_{p}\right)$. We shall now show that if $G_{2}=G / C^{*}$ then it suffices to prove that $G_{2} / O_{p}\left(G_{2}\right)$ is abelian and is of exponent dividing $p-1$ which in fact implies that $G_{1} / O_{p}\left(G_{1}\right)$ possesses the same properties.

It may be observed that $\bar{Q}_{p}$ can be considered as a vector space over $\mathrm{GF}(p)$ and the group of automorphisms induced by $G$ in $\bar{Q}_{p}$, i.e. $G / C^{*}=$ $G_{2}$, can be regarded as a group of linear transformations of $\bar{Q}_{p}$.

Since $C_{1}=C^{*} / C$ is a group of automorphisms that induces identity on $\bar{Q}_{p}$ it follows that $\left|C_{1}\right|$ is a power of $p$ and hence the normal $p$-subgroup $C_{1}$ of $G_{1}$ is contained in $O_{p}\left(G_{1}\right)$. Also, if $O_{p}\left(G_{2}\right)=L / C_{1}$ then it implies that $L$ is a normal $p$-subgroup of $G_{1}$ and therefore $L \subseteq O_{p}\left(G_{1}\right)$. Thus $O_{p}\left(G_{2}\right)=O_{p}\left(G_{1}\right) / C_{1}$ and $G_{2} / O_{p}\left(G_{2}\right)$ is isomorphic to $G_{1} / O_{p}\left(G_{1}\right)$. As a first step towards proving $G_{2} / O_{p}\left(G_{2}\right)$ is abelian and is of exponent dividing $p-1$ we shall show that $O_{p}\left(G_{2}\right)$ possesses the same properties.

From Lemma 1.4 in [3] we know that the Sylow $p$-subgroup of the quasicenter is generated by QC-elements and this implies that there exists QC-elements $x_{1}, x_{2}, \cdots, x_{n}$ such that $B=\left\{\bar{x}_{1}, \bar{x}_{2}, \cdots, \bar{x}_{n}\right\}$ is a basis for $\bar{Q}_{p}$ where $\bar{x}_{i}=x_{i} \Phi\left(Q_{p}\right)$. Now, if $y$ is a $p^{\prime}$-element of $G$ then $\left\langle x_{i}\right\rangle\langle y\rangle=\langle y\rangle\left\langle x_{i}\right\rangle$ implies that $y$ normalises $\left\langle x_{i}\right\rangle$. (This follows from the fact that $\left\langle x_{i}\right\rangle$ is a Sylow $p$-subgroup of the quasicenter of $\left.S=\left\langle x_{i}\right\rangle\langle y\rangle=\langle y\rangle\left\langle x_{i}\right\rangle.\right)$ Therefore $\bar{y}=y \Phi\left(Q_{p}\right)$ normalises $\bar{x}_{i}$. But $\Phi\left(Q_{p}\right) \subseteq C_{G}\left(Q_{p} / \Phi\left(Q_{p}\right)\right)=C^{*}$ and therefore $G_{2}=G / C^{*}$ is a homomorphic image of $\bar{G}$ under the natural homomorphism $\theta$ of $\bar{G}=G / \Phi\left(Q_{p}\right)$ to $G_{2}=G / C^{*}$. Therefore, if $\hat{y}$ is the image of $\bar{y}$ under $\theta$ then $\hat{y}$ shall send every basis element $\bar{x}_{i}$ to $s \bar{x}_{i}$ (using additive notation in $\bar{Q}_{p}$ ) where $s \in \operatorname{GF}(p)$. Therefore, the matrix of $\hat{y}$ is diagonal and $\hat{y}^{p-1}=1$ since the multiplicative group of $\mathrm{GF}(p)$ is of order $p-1$ and the product of diagonal matrices is again a diagonal matrix. Since the product of two diagonal matrices commutes it follows that the totality of $p^{\prime}$-elements forms an abelian subgroup of exponent dividing $p-1$, i.e., $O^{p}\left(G_{2}\right)$ is abelian and is of exponent dividing $p-1$. If $p=2$ then $O^{p}\left(G_{2}\right)=1$ and $G_{2}$ is a 2-group. But then $G_{2} / O_{p}\left(G_{2}\right)$ is trivially abelian and is of exponent dividing $p-1$. Thus we may assume that $p>2$. Let $\bar{Q}_{p}=V_{0} \supset V_{1} \supset V_{2} \supset \cdots \supset V_{n}=0$ be a $G_{2}$ composition series for $\bar{Q}_{p}$ and let $K_{i}=C_{G_{2}}\left(V_{i} / V_{i+1}\right), 0 \leqq i \leqq n-1$. It is 
evident from the above discussion that $O^{p}\left(G_{2} / K_{i}\right)$ is abelian and is of exponent dividing $p-1$. We now show that every $p$-element of $G_{2} / K_{i}$ has $(x-1)^{2}$ as minimum polynomial.

Let $y$ be a $p$-element of $G$ and $H_{i}=\left\langle x_{i}\right\rangle\langle y\rangle=\langle y\rangle\left\langle x_{i}\right\rangle \forall i$. Then $H_{i} \cap Q_{p}=$ $\left\langle x_{i}\right\rangle \cdot\left(\langle y\rangle \cap Q_{p}\right)$ and $\left(H_{i} \cap Q_{p}\right)^{y} \subseteq H_{i}^{v} \cap Q_{p}^{y}=H_{i} \cap Q_{p}$. Thus $H_{i} \cap Q_{p}$ is normalised by $y$ so that $\bar{y}=y \Phi\left(Q_{p}\right)$ normalises $\left(H_{i} \cap Q_{p}\right)^{*}$. Hence the subspace $\left(H_{i} \cap Q_{p}\right)^{*}$ of $\bar{Q}_{p}$ is invariant under $\hat{y}=y C^{*}$. $(\hat{y}$ is not the identity transformation.) If the order of $y$ is $p^{m}$ then $\hat{y}^{p^{m}}=1$, i.e. $\hat{y}^{p^{m}}-1=0$. This implies that the linear transformation $\hat{y}$ is annulled by the polynomial $x^{p^{m}}-1=(x-1)^{p^{m}}$ and since the dimension of $\left(H_{i} \cap Q_{p}\right)^{*}$ is at most 2 it follows that the minimum polynomial of $\hat{y}$ is $(x-1)^{2}$ whence $(\hat{y}-1)^{2}=0$. Since every $p$-element of $G_{2} / K_{i}$ is of the form $\hat{y} K_{i}$ where $\hat{y}$ is a $p$-element of $G_{2}$ it follows that every $p$-element of $G_{2} / K_{i}$ has $(x-1)^{2}$ as minimum polynomial. Also, the Sylow 2-subgroup of $G_{2} / K_{i}$ being contained in $O^{p}\left(G_{2} / K_{i}\right)$ is abelian and hence Theorem B of [2] (Hall-Higman) applies and we conclude that $G_{2} / K_{i}$ is abelian and is of exponent dividing $p-1$. But this again implies that $G_{2} / \bigcap_{i=0}^{n-1} K_{i}$ is abelian and is of exponent dividing $p-1$, i.e. $G_{2} / O_{p}\left(G_{2}\right)$ is abelian and is of exponent dividing $p-1$. The theorem is therefore proved completely.

The supersolvability of the hyperquasicenter enables one to characterize supersolvable groups in certain special ways and the following theorems which are quite easy to prove may be mentioned in this connection.

THEOREM. A group $G$ is supersolvable if and only if, for every subgroup $S=H_{1} H_{2}\left(H_{1}\right.$ and $H_{2}$ are subgroups of $\left.S\right), Q^{*}(S)=Q^{*}\left(H_{1}\right) \cdot Q^{*}\left(H_{2}\right)$.

THEOREM. A subgroup $G$ is supersolvable if and only if $H \cap Q^{*}(G)=$ $Q^{*}(H)$ for every subgroup $H$ of $G$.

The author would like to record his deep gratitude to the referee of this paper for his invaluable help in its preparation.

\section{BIBLIOGRAPHY}

1. O. Ore, Contributions to the theory of groups of finite order, Duke Math. J. 5 (1939), 431-460.

2. P. Hall and Graham Higman, On the p-length of p-soluble groups and reduction theorems for Burnside's problem, Proc. London Math. Soc. (3) 6 (1956), 1-42. MR 17, 344.

3. N. P. Mukherjee, The hyperquasicenter of a finite group. I, Proc. Amer. Math. Soc. 26 (1970), 239-243.

4. R. Baer, Principal factors, maximal subgroups and conditional identities of finite groups, Illinois J. Math. 13 (1969), 1-52. MR 38 \#5933.

Department of Mathematics, West Virginia University, Morgantown, West VIRGINIA 26506 